\title{
Kohler-Spiegel, Helga
}

"Doing Gender" lernen. Geschlechtergerechte Bildung im Religionsunterricht Zeitschrift für Pädagogik und Theologie 72 (2020) 1, S. 66-78

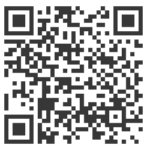

Quellenangabe/ Reference:

Kohler-Spiegel, Helga: "Doing Gender" lernen. Geschlechtergerechte Bildung im Religionsunterricht - In: Zeitschrift für Pädagogik und Theologie 72 (2020) 1, S. 66-78 - URN:

urn:nbn:de:0111-pedocs-213939 - DOI: 10.25656/01:21393

https://nbn-resolving.org/urn:nbn:de:0111-pedocs-213939

https://doi.org/10.25656/01:21393

in Kooperation mit / in cooperation with:

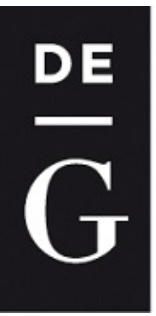

\section{DE GRUYTER}

https://www.degruyter.com

\section{Nutzungsbedingungen}

Gewährt wird ein nicht exklusives, nicht übertragbares, persönliches und beschränktes Recht auf Nutzung dieses Dokuments. Dieses Dokument ist ausschließlich für den persönlichen, nicht-kommerziellen Gebrauch bestimmt. Die Nutzung stellt keine Übertragung des Eigentumsrechts an diesem Dokument dar und gilt vorbehaltlich der folgenden Einschränkungen: Auf sämtlichen Kopien dieses Dokuments müssen alle Urheberrechtshinweise und sonstigen Hinweise auf gesetzlichen Schutz beibehalten werden. Sie dürfen dieses Dokument nicht in irgendeiner Weise abändern, noch dürfen Sie dieses Dokument für öffentliche oder kommerzielle Zwecke vervielfältigen, öffentlich ausstellen, aufführen, vertreiben oder anderweitig nutzen.

Mit der Verwendung dieses Dokuments erkennen Sie die Nutzungsbedingungen an.

\section{Terms of use}

We grant a non-exclusive, non-transferable, individual and limited right to using this document.

This document is solely intended for your personal, non-commercial use. Use of this document does not include any transfer of property rights and it is conditional to the following limitations: All of the copies of this documents must retain all copyright information and other information regarding legal protection. You are not allowed to alter this document in any way, to copy it for public or commercial purposes, to exhibit the document in public, to perform, distribute or otherwise use the document in public.

By using this particular document, you accept the above-stated conditions of use.

\section{Kontakt / Contact:}

\section{peDOcs}

DIPF | Leibniz-Institut für Bildungsforschung und Bildungsinformation Informationszentrum (IZ) Bildung

E-Mail: pedocs@dipf.de

Internet: www.pedocs.de

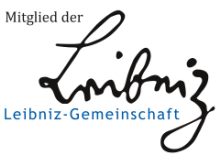




\section{ZEITSCHRIFT FÜR PÄDAGOGIK UND THEOLOGIE}

HERAUSGEBER

David Käbisch, Frankfurt/Main

Ralf Koerrenz, Jena

Martina Kumlehn, Rostock

Thomas Schlag, Zürich

Friedrich Schweitzer, Tübingen

Henrik Simojoki, Bamberg

KORRESPONDIERENDE HERAUSGEBER

Frauke Büchner, Landolfshausen

Michael Domsgen, Halle/Saale

Bernhard Dressler, Marburg

Volker Elsenbast, Landau

Heid Leganger-Krogstad, Oslo

Boguslaw Milerski, Warschau

Dávid Németh, Budapest

Kati Tervo-Niemelä, Helsinki

Uta Pohl-Patalong, Kiel

Henning Schlu $\beta$, Wien

Bernd Schröder, Göttingen

Andrea Schulte, Erfurt

Athanasios Stogiannidis, Thessaloniki

Dietrich Zilleßen, Bergisch Gladbach

\section{DE GRUYTER}


ABSTRACTED/INDEXED IN Baidu Scholar - CNKI Scholar (China National Knowledge Infrastructure) · CNPIEC: cnpLINKer - Dimensions · EBSCO Discovery Service · Google Scholar · IBR (International Bibliography of Reviews of Scholarly Literature in the Humanities and Social Sciences) · IBZ (International Bibliography of Periodical Literature in the Humanities and Social Sciences) $\cdot$ Index Theologicus · J-Gate $\cdot$ JournalTOCs $\cdot$ KESLI-NDSL (Korean National Discovery for Science Leaders) - Microsoft Academic $\cdot$ MyScienceWork · Naver Academic · Naviga (Softweco) · Primo Central (ExLibris) · Publons · QOAM (Quality Open Access Market) · ReadCube · Semantic Scholar · Summon (ProQuest) · TDNet · Ulrich's Periodicals Directory/ulrichsweb · WanFang Data · WorldCat (OCLC)

ISSN $1437-7160 \cdot$ e-ISSN 2366-7796

Alle Informationen zur Zeitschrift, wie Hinweise für Autoren, Open Access, Bezugsbedingungen und Bestellformulare, sind online zu finden unter www.degruyter.com/journals/zpt

VeRANTWORTLICHE HeRAUSGebeR Prof. Dr. David Käbisch, Fachbereich Ev. Theologie, Goethe-Universität, Norbert-Wollheim-Platz 1, 60323 Frankfurt, kaebisch@em.uni-frankfurt.de; Prof. Dr. Dr. Ralf Koerrenz, Institut für Bildung und Kultur der Friedrich-Schiller-Universität Jena, Am Planetarium 4, 07743 Jena, ralf.koerrenz@uni-jena.de;

Prof. Dr. Martina Kumlehn, Theologische Fakultät, Universitätsplatz 1, 18055 Rostock, martina.kumlehn@uni-rostock.de;

Prof. Dr. Thomas Schlag, Theologische Fakultät, Kirchgasse 9, CH-8001 Zürich, thomas.schlag@theol.uzh.ch;

Prof. Dr. Dr. h.c. Friedrich Schweitzer, Universität Tübingen, Evangelisch-Theologische Fakultät, Liebermeisterstr. 12, 72076 Tübingen, friedrich.schweitzer@uni-tuebingen.de;

Prof. Dr. Henrik Simojoki, Institut für Evangelische Theologie, Otto-Friedrich-Universität Bamberg, An der Universität 11, 96047 Bamberg, henrik.simojoki@uni-bamberg.de;

Betreuung Rezensionswesen: Prof. Dr. Thomas Schlag

JOURNAL MANAGER Claudia Hill, De Gruyter, Genthiner Straße 13, 10785 Berlin, Germany, Tel.: +49 (0)30 26005 - 172, Fax: +49 (0)30 26005 - 250,

E-Mail: claudia.hill@degruyter.com

ANZEIGENVERANTWORTLICHE Claudia Neumann, De Gruyter, Genthiner Straße 13, 10785 Berlin, Germany. Tel.: +49 (0)30 260 05-226, Fax: +49 (0) 30260 05-264,

E-Mail: anzeigen@degruyter.com

(C) 2020 Walter de Gruyter GmbH, Berlin/Boston

SATZ Dörlemann Satz, Lemförde

DRUCK Franz X. Stückle Druck und Verlag e.K., Ettenheim

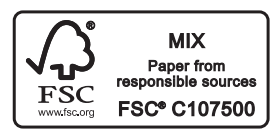




\section{Inhaltsverzeichnis}

\section{Themenheft: Gender}

Editorial - 1

Martina Kumlehn und Thomas Schlag

\section{Beiträge}

Renate Wieser

Die „Sache mit dem Geschlecht": Zum Stand der Debatte — 4

Gero Bauer, Sebastian Engelmann

Queer(ed) Pedagogies: A Critical Perspective - 18

Nina Degele

Der schwangere Arzt im Praktikum: Interventionen der Gender Studies zu geschlechtergerechter Sprache - 30

Peter-Ben Smit

Das Thema Männlichkeit im hochschuldidaktischen Verwendungszusammenhang - am Beispiel der theologischen Lehre im Fachbereich Neues Testament -42

Annebelle Pithan, Uta Pohl-Patalong, Susanne Schwarz

Zwischen „Gewordensein und Noch-Nicht““. Ein Schreibgespräch zur

Entwicklung der Genderthematik in der Religionspädagogik — 55

Helga Kohler-Spiegel

„Doing Gender“ lernen. Geschlechtergerechte Bildung im

Religionsunterricht - 66

Fahimah Ulfat

Sexualität und Religion bei jungen Muslim*innen in Deutschland in islamisch-religionspädagogischer Perspektive -79 


\section{Das besondere Buch}

Ralf Koerrenz

Samuel Salzborn, Globaler Antisemitismus. Eine Spurensuche in den Abgründen der Moderne - 96

\section{Rezensionen}

\section{Manfred Pirner}

Hyun-Sook Kim, Richard R. Osmer und Friedrich Schweitzer: The Future of Protestant Religious Education in an Age of Globalization — 103

Peter Schreiner

Jenny Berglund (Ed.), European Perspectives on Islamic Education and Public Schooling — 106

\section{Friedrich Schweitzer}

Weilert, A. Katarina/Hildmann, Philipp W. (Hg.): Religion in der Schule.

Zwischen individuellem Freiheitsrecht und staatlicher Neutralitätsverpflichtung — 110

Reinhold Mokrosch

Michael Fricke, Lothar Kuld, Anne Sliwka (Hg.): Konzepte sozialer Bildung an der Schule. Compassion - Diakonisches Lernen - Service Learning — 113 


\title{
Helga Kohler-Spiegel* \\ „Doing Gender" lernen. \\ Geschlechtergerechte Bildung im \\ Religionsunterricht
}

https://doi.org/10.1515/zpt-2020-0007

\begin{abstract}
Doing gender“ takes account of the social construction of gender. Here, gender is not considered an attribute or characteristic of an individual; it is rather a social and cultural construct which is constantly staged, produced, and reproduced. „Doing gender“ in an academic context concerns the teachers' basic understanding of and attitude towards the topic of gender, as well as the education system as a whole. This basic understanding also has to be applied in religious education in order to guarantee learning free of gender bias in religious studies. Some examples complete the article.
\end{abstract}

Zusammenfassung: „Doing gender“ nimmt die soziale Konstruktion von Geschlecht in den Blick. Geschlecht bzw. Geschlechtszugehörigkeit werden nicht als Eigenschaft oder Merkmal von Individuen verstanden, sondern sie sind sozial und kulturell konstruiert und in Interaktionen ständig inszeniert, produziert und reproduziert. Doing gender im Schulbereich betrifft das Grundverständnis und die Haltung der Lehrpersonen zum Thema Geschlecht sowie die Institution Schule insgesamt. Dieses Grundverständnis ist auch im Religionsunterricht umzusetzen, um geschlechtergerechte Bildung im religiösen Bereich zu gewährleisten. Beispiele dazu runden den Beitrag.

Keywords: Doing gender, reflecting gender, gender oriented learning, religious studies

Schlagworte: Doing gender, Reflexion von Geschlecht, geschlechtergerechte Bildung, Religionsunterricht

„Beim Lehrausgang zur Bergkirche hatten wir einen anspruchsvollen Weg zu bewältigen. Auch die Mädchen haben ihn gut gemeistert.“ „Überraschend war, wie aufmerksam die Jungen der Erzählung von Mose zugehört haben. Das hat sicher damit zu tun, dass es eine spannende Geschichte ist.“ „Wo ist Klaus? Ach, er hat

\footnotetext{
*Kontakt: Dr. Helga Kohler-Spiegel, Fachbereich Human- und Bildungswissenschaften, Pädagogische Hochschule Vorarlberg, Liechtensteiner Straße 33-37, A-6800 Feldkirch, E-Mail: h.kohler-spiegel@ksnet.at
} 
sich bei den Mädchen versteckt.“ Diese Aussagen von Lehrpersonen zeigen deren geschlechtsspezifische Wahrnehmung sowie die damit verbundene Erwartung an das Verhalten der Schülerinnen und Schüler und die Bewertung dieses Verhaltens. Zugleich prägen diese Zuschreibungen die Schülerinnen und Schüler.

\section{Vergewisserung: Geschlecht und Gender}

Lange Zeit galt Geschlecht als etwas Eindeutiges und von Geburt an Irreversibles; Verhaltensweisen und Verhaltensdeutungen werden vorbewusst von der Geschlechtszugehörigkeit bestimmt. Carol Hagemann-White ${ }^{1}$ nannte es ein „kulturelles System der Zweigeschlechtlichkeit“2. Die Unterscheidung zwischen Sex und Gender schaffte das Bewusstsein, dass biologische Differenz zwischen den Geschlechtern keine soziale Ungleichheit rechtfertigt, dass Gleichheit und Differenz miteinander zu denken sind. „Sex“ meint die Geburtsklassifikation des körperlichen Geschlechts aufgrund vereinbarter biologischer Kriterien. „Gender“ beschreibt das soziale und kulturelle Geschlecht als soziale Konstruktion, das durch normative Zuschreibungen auf der Basis des biologischen Geschlechts bestimmt ist. ${ }^{3}$

Candace West und Don Zimmerman ${ }^{4}$ fügten eine dritte Kategorie hinzu: „,sex category“. Sex category meint „die soziale Zuordnung zu einem Geschlecht im Alltag aufgrund der sozial geforderten Darstellung einer erkennbaren Zugehörigkeit zur einen oder anderen Kategorie. Diese muss der Geburtsklassifikation nicht entsprechen.“"5

1 Vgl. Carol Hagemann-White, Sozialisation: Weiblich - männlich?. Opladen (Leske und Budrich) 1984, 78-85.

$2 \mathrm{Zu}$ den Modellen der Verhältnisbestimmung zwischen den Geschlechtern vgl. exemplarisch: Gisela Matthiae, Von der Emanzipation über die Dekonstruktion zur Restauration und zurück. Genderdiskurse und Geschlechterverhältnisse. In: Annabelle Pithan u.a. (Hg.), Gender - Religion - Bildung. Beiträge zu einer Religionspädagogik der Vielfalt. Gütersloh (Gütersloher Verlagshaus) 2009, 30-46.

3 Vgl. Regina Becker-Schmidt/Gudrun-Axeli Knapp, Feministische Theorien zur Einführung. Hamburg (Junius) 2003, 69.

4 Vgl. Candace West/Don H. Zimmerman: Doing Gender. In: Gender and Society 1 (1987), Nr. 2, 125-151, 131 ff. (online abrufbar unter http://www.csun.edu/ snk1966/West\%20and\%20 Zimmerman\%20Doing\%20Gender.pdf, Lesedatum: 12. September 2019).

5 Die Gleichstellungsbeauftragte, Genderportal (online abrufbar unter https://www.uni-due.de/ genderportal/lehre_erwachsenenbildung_doinggender.shtml, Lesedatum: 12. September 2019). 
“Doing gender is unavoidable.“6 „Doing gender“ beschreibt den (inter-) aktiven Charakter der Produktion und Reproduktion von Geschlecht und Geschlechterdifferenz, es wird in der sozialen Interaktion konstruiert und strukturiert zugleich die Interaktionen. ${ }^{7}$ "Doing gender involves a complex of socially guided perceptual, interactional, and micropolitical activities that cast particular pursuits as expressions of masculine and feminine ,nature“." 8 Geschlecht ist also keine Zuschreibung, sondern eine soziale Konstruktion, ein „Tun”, das in den Handlungen ständig entwickelt und verändert wird. Harold Garfinkel dokumentierte, wie Agnes sich im Verlauf ihrer Geschlechtsumwandlung die Repertoires von Weiblichkeit aneignete und wie Geschlecht im Alltag inszeniert, gestaltet und verändert wird..$^{9}$ Aufgrund des wahrgenommenen Geschlechts entstehen Erwartungen an das Gegenüber bzw. Aussehen und Verhalten oder es werden unterschiedliche Interessen oder unterschiedliche kognitive, emotionale oder motorische Fähigkeiten zugeschrieben. Erlebt wird das Geschlecht aber meist nicht als Produkt der interaktiven Konstruktion, sondern ,als natürlich vorgegeben“ und/ oder „in der Erziehung erworben“. Candace West und Sarah Fenstermaker haben 1995 mit dem Konzept von „Doing Difference“ den Ansatz von Doing Gender weiterentwickelt, indem sie zusätzlich zu „gender“ die Kategorien „class - soziale Klasse“ und „race - ethnische Zugehörigkeit“ eingeführt haben. ${ }^{10}$ Stefan Hirschauer verweist mit „Un-doing Gender“ darauf, dass es soziale Situationen gibt, in denen Geschlecht zwar registriert wird, aber keine hervorgehobene Bedeutung hat. $^{11}$

6 West/Zimmerman, Doing Gender, 137.

7 Vgl. Alexander Geimer, Doing Gender (nach West/Zimmerman) (online abrufbar unter https:// gender-glossar.de/glossar/archivbeitraege-alphabetisch/item/18-doing-gender, Lesedatum: 13. September 2019).

8 West/Zimmerman, Doing Gender, 126.

9 Vgl. Harold Garfinkel, Studies in Ethnomethodology. Cambridge (Polity Press) 1984.

10 Vgl. Candace West/Sarah Fenstermaker, Doing difference. In: Gender and Society 9 (1995), Nr. 1, 8-37, $14 \mathrm{f}$.

11 Vgl. Stefan Hirschauer, Un/doing Differences. Die Kontingenz sozialer Zugehörigkeiten. Un/doing Differences. The Contingency of Social Belonging. In: Zeitschrift für Soziologie 43 (2014), Nr. 3, 170-191, 182 ff. (online abrufbar unter https://www.degruyter.com/downloadpdf/j/ zfsoz.2014.43.issue-3/zfsoz-2014-0302/zfsoz-2014-0302.pdf, Lesedatum: 12. September 2019). 


\section{Entwicklungsbezogene Aspekte}

Neben den häufig bedachten Faktoren geschlechtsspezifischer Sozialisation ${ }^{12}$ sind auch entwicklungspsychologische Aspekte im Blick auf Gender für altersentsprechendes Arbeiten zu bedenken. Auch hier gilt es, sich von Biologismen zu lösen und „Geschlecht“", das meist als „Natur“ erlebt wird, als sozial konstruiert zu verstehen. Ein paar Schlaglichter:

Mit ca. drei Jahren besitzen Kinder eine ausgeprägte Geschlechtsidentität, die sie auch nach außen verteidigen. Ihre Vorstellungen von Weiblichkeit und Männlichkeit sind relativ umfassend und stark stereotyp, aber noch wenig mit Wertungen verbunden. Die Erziehung in Familie und den öffentlichen Einrichtungen fördert häufig die aktiv-aggressive Entfaltung von Jungen, während Mädchen besonders in familiale und soziale Aufgaben mit geringerem Bewegungsspielraum eingebunden werden. ${ }^{13}$

Überraschend das Ergebnis einer Untersuchung „Sozialverhalten von Vorschulkindern" von Horst Nickel und Ulrich Schmidt-Denter von 1980, bei der über 400 Kinder im Alter von drei bis fünf Jahren aus traditionell geführten Kindergärten und aus Kinderläden beforscht wurden. Die Dominanz der Jungen über die drei- bis vierjährigen Mädchen war eindrücklich. Je weniger Struktur vorgegeben ist, desto stärker sind die Dominanz der Jungen und der Rückzug der Mädchen. „In den Kinderläden ... traten die Mädchen signifikant häufiger den Rückzug an und wurden von den Jungen massiv dominiert. “14 Jungen reagieren stärker auf die Frage, wer hier der Chef ist, Erwachsene müssen als „Rudelchef/in“ akzeptiert sein, der bzw. die die Spielregeln nicht nur vorgibt, sondern auch einfordert. Dies erfordert klare Führung statt Diskussion und Bitten, und den Mut, sich den Machtkämpfen zu stellen. ${ }^{15}$

12 Vgl. exemplarisch Marianne Grabrucker, „Typisch Mädchen ...“ Prägung in den ersten drei Lebensjahren. Ein Tagebuch. Frankfurt a.M. (Fischer) 1985.

13 Vgl. Karin Flaake/Vera King (Hg.), Weibliche Adoleszenz. Zur Sozialisation junger Frauen. Frankfurt (Springer) ${ }^{2} 1993$. Vgl. exemplarisch: Helga Kohler-Spiegel, Gender im Religionsunterricht - Mädchen/Jungen im Religionsunterricht. In: Christoph Bizer u. a. (Hg.), Religionsdidaktik (=Jahrbuch der Religionspädagogik 18). Neukirchen-Vluyn (Neukirchener) 2002, 157-170.

14 Doris Bischof-Köhler, Geschlechtstypisches Verhalten von Jungen aus evolutionstheoretischer und entwicklungspsychologischer Perspektive. In: Michael Matzner/Wolfgang Tischner (Hg.), Handbuch Jungen-Pädagogik, Weinheim (Beltz) 2008, 18-33, $20 \mathrm{f.}$

15 Vgl. Thorsten Knauth, Jungen in der Religionspädagogik. Bestandaufnahme und Perspektiven. In: Annabelle Pithan u. a. (Hg.), Gender - Religion - Bildung. Beiträge zu einer Religionspädagogik der Vielfalt. Gütersloh (Gütersloher Verlagshaus) 2009, 72-94. Vgl. Markus Hofer, Versteh eine die Jungs. Gebrauchsanweisung für Buben und Burschen. Broschüre der katholischen Kirche Feldkirch 2012. Vgl. Reinhard Winter, Jungen - eine Gebrauchsanweisung. Jungen verstehen und unterstützen, Weinheim (Beltz) 2011. 
Im Schulalter und in der Pubertät rückt das subjektive Körpergefühl in den Mittelpunkt des Interesses der Heranwachsenden. Jungen holen sich dies teilweise über Raufen, körperbetonte Spiele und körperbetonte Sportarten. Zur Orientierung ist bei Jungen die Clique wichtig, der Platz in der Gruppe. ${ }^{16}$ Beide, Mädchen wie Jungen, müssen mit Nähe und Distanz experimentieren dürfen, Zuwendung zeigen können, ohne dass sie missbraucht wird. Für Mädchen spielt die enge und dauerhafte Beziehung zu einer gleichgeschlechtlichen Person eine stärkere Rolle als Peers. Untersuchungen ergaben, dass die Mädchenfreundschaften wichtige Stabilisierungsfunktion haben, sie geben emotionalen Halt im Klassenverband und schützen vor Diskriminierung. „Alles zusammen machen“ stärkt das Selbstwertgefühl, Mädchen sichern sich so gegenseitig bzgl. ihrer Gefühle und ihres Handelns ab. ${ }^{17}$

\section{Doing Gender - ein Kinderrecht}

Es ist ein Recht von Kindern, sich ohne Diskriminierung, im Bewusstsein von Geschlecht und zugleich unabhängig von ihrem Geschlecht entwickeln zu dürfen. Kinderrechtsorientierte Pädagogik ${ }^{18}$ versteht sich so, dass pädagogische Konzeptionen und pädagogisches Handeln dem Recht des Kindes auf ein Leben ohne Diskriminierung, unter Berücksichtigung des Kindeswohls und des Kindeswillens verpflichtet sein müssen. Schulkultur und schulisches Handeln werden daran geprüft, ob sie Leben und Lernen in der Schule ohne Diskriminierung und unter Beteiligung aller Kinder umsetzen. In den Leitbildern der Schulen wird sichtbar,

16 Vgl. Melitta Walter, Jungen sind anders, Mädchen auch. Den Blick schärfen für eine geschlechtergerechte Erziehung. München (Kösel) 2005. Vgl. Barbara Rendtorff/Vera Mosera (Hg.), Geschlecht und Geschlechterverhältnisse in der Erziehungswissenschaft. Eine Einführung. Opladen (VS Verlag für Sozialwissenschaften) u.a. 1999.

17 Vgl. Karin Flaake, Geschlechterverhältnisse - Adoleszenz - Schule. Männlichkeits- und Weiblichkeitsinszenierungen als Rahmenbedingungen für pädagogische Praxis. Benachteiligte Jungen und privilegierte Mädchen? Tendenzen aktueller Debatten. In: Sabine Jösting/Malwine Seemann (Hg.), Gender und Schule. Geschlechterverhältnisse in Theorie und schulischer Praxis. Oldenburg (BIS-Verlag der Carl von Ossietzky Universität Oldenburg) 2006, 27-44, 34 ff.

18 Vgl. Helga Kohler-Spiegel, „Kinder haben Rechte, das gilt für jedes Kind ...“. Kinderrechte. In: Stefan Altmeyer u. a. (Hg.), Menschenrechte und Religionsunterricht (= Jahrbuch Religionspädagogik 33). Göttingen (Vandenhoeck und Ruprecht) 2017, 153-161. Vgl. Bundesministerium für Familie, Senioren, Frauen und Jugend (Hg.), Übereinkommen über die Rechte des Kindes. UN-Kinderrechtskonvention im Wortlaut und Materialien. Berlin 2007, 87. 
welche Kinderrechte im Vordergrund stehen, wie Heterogenität ${ }^{19}$, Diversität und Gender in der Schule umgesetzt werden, wie Nicht-Diskriminierung, Gleichbehandlung und Inklusion unter den Geschlechtern ebenso wie unter den Kulturen, den Religionen und den Stärken und Handicaps von Kindern als „Pädagogik der Vielfalt“ ${ }^{20}$ schulisch gelebt wird.

\section{Doing Gender - Religionsunterricht im System Schule}

Eine solche kinderrechtsorientierte Pädagogik der Vielfalt findet im Rahmen von Schule statt. Die jeweilige Erwachsenengeneration legt fest, welche Bildungsmöglichkeiten und Bildungsverpflichtungen der nächsten Generation zur Verfügung stehen, welche Kompetenzen junge Menschen erwerben und mit welchen Inhalten sie sich beschäftigen sollen. Der Religionsunterricht ist Teil von Schule mit ihrer Qualifikations-, Integrations- und Selektionsfunktion. Die „zehn Merkmale für guten Unterricht“ nach Hilbert Meyer gelten auch für den Religionsunterricht: klare Strukturierung des Unterrichts, hoher Anteil echter Lernzeit, lernförderliches Klima, inhaltliche Klarheit, sinnstiftende Kommunikation, Methodenvielfalt, individuelles Fördern, intelligentes Üben, transparente Leistungserwartungen und vorbereitete Umgebung. ${ }^{21}$

Nach John Hattie (815 Metaanalysen mit über 50.000 Studien) ${ }^{22}$ ist es nicht einfach die Lehrperson, die zählt, sondern das Handeln, das Tun der Lehrperson: Entscheidend ist, dass die Lehrperson an den Schülerinnen und Schülern

19 Vgl. exemplarisch: Bernhard Grümme, Heterogenität (online abrufbar unter https://www. bibelwissenschaft.de/wirelex/das-wissenschaftlich-religionspaedagogische-lexikon/lexikon/ sachwort/anzeigen/details/heterogenitaet/ch/1e56e145b8d883647582cbbeac9c2dcf/, Lesedatum: 12. September 2019).

20 Vgl. Annedore Prengel, Pädagogik der Vielfalt. Verschiedenheit und Gleichberechtigung in Interkultureller, Feministischer und Integrativer Pädagogik. Opladen u. a. (VS Verlag für Sozialwissenschaften) ${ }^{3} 2006$.

21 Vgl. Helga Kohler-Spiegel, Was ist guter Unterricht? In: Katechetische Blätter 132 (2007), 238-240. Vgl. Rudolf Englert u.a. (Hg.), Was ist guter Religionsunterricht? (= Jahrbuch Religionspädagogik 22). Neukirchen-Vluyn (Neukirchener) 2006.

22 John Hattie, Visible Learning. A Synthesis of Over 800 Meta-Analyses Relating to Achievement, Abingdon/GB 2009, übersetzt und bearbeitet von Wolfgang Beywl/Klaus Zierer, John Hattie, Lernen sichtbar machen. Baltmannsweiler (Schneider) 2013, v. a. 27 bis 46. Vgl. auch: SQA Schulqualität Allgemeinbildung/Bundesministerium für Unterricht, Kunst und Kultur (Hg.), Die HattieStudie. Wien 2012, 5. 
interessiert ist, dass sie auf ein vertrauensvolles, angstfreies, fehlerfreundliches und menschlich zugewandtes Miteinander verbunden mit hohen Ansprüchen an Einsatz und Anstrengungsbereitschaft achtet. ${ }^{23}$

\subsection{Handeln und Haltung der Lehrperson zählen}

Die Person des Lehrers, der Lehrerin löst Reaktionen bei Schülerinnen und Schülern aus. So müssen beispielsweise weibliche Lehrpersonen im Grundschulbereich einschreiten und Verhalten von Schülerinnen und Schülern korrigieren, das mit Übertragungen von Verhalten der eigenen Mutter gegenüber zu tun hat. Lehrpersonen lösen Übertragungen aus - als Mann und als Frau, mütterlich, väterlich ... Heute wird zwischen spontanen Übertragungen und den klassischen „Übertragungen“ unterschieden, die Sigmund Freud „eine Verwechslung in der Zeit“ genannt hat. In der Schule spielen die spontanen Übertragungen eine wichtige Rolle, aber auch „Verwechslungen in der Zeit“ können vorkommen. ${ }^{24} \mathrm{Er}$ fahrungen mit eigenen Eltern und Betreuungspersonen werden auf Lehrerinnen und Lehrer übertragen, dies kann teilweise die schulischen Beziehungen stärken, teilweise auch belasten.

Auch seitens der Lehrperson ist die Reflexion eigener biografischen Erfahrungen, Sozialisation und Prägungen notwendig. Solche Prägungen zeigen sich häufig in „Glaubenssätzen“, d. h. verinnerlichte Zuschreibungen, die das Denken und Handeln einer Person (oft unbewusst) beeinflussen, z.B: „Du bist nur gut, wenn Du arbeitest.“ „Du bist nur gut, wenn du für andere da bist.“ „Du musst immer stark sein.“ Solche Zuschreibungen und Prägungen werden in Interaktionen immer wieder geschlechtsbezogen aktiviert. Die eigene Glaubensbiografie und die Sozialisation als Religionslehrperson können diese Prägungen verstärken. $^{25}$

23 Vgl. Rudolf Englert, Der Religionsunterricht im Lichte der Hattie-Studie. In: Katechetische Blätter 138 (2013), 444-450.

24 Vgl. Helga Kohler-Spiegel, Gesehen werden - gebunden sein. Ein psychologischer Blick auf eine „Kultur der Anerkennung“. In: Thomas Krobath u.a. (Hg.), Anerkennung in religiösen Bildungsprozessen. Interdisziplinäre Perspektiven (= Wiener Forum für Theologie und Religionswissenschaft, 8). Göttingen (Vandenhoeck und Ruprecht) 2013, 103-113.

25 Vgl. Helga Kohler-Spiegel, In der Schule leben und überleben. In: Ludwig Rendle (Hg.), Ganzheitliche Methoden in der Schulpastoral. München (Kösel) 2013, 34-44. 


\subsection{Wen benachteiligt das Schulsystem?}

Gegenwärtig ist vermehrt von einer Benachteiligung der Jungen im Schulsystem die Rede. In der Süddeutschen Zeitung vom 7.1.2013 heißt es: „Wenn Jungs sich in der Schule wie Jungs benehmen, gelten sie als verhaltensauffällig. “26 Der höhere Bewegungsdrang, der bei Jungen zu beobachten ist, findet im Arbeitsrhythmus (kurze Bewegungsübungen, Toilette oder Wassertrinken) oder methodisch (Laufaufgaben, Werkstatt, Stationen ...) Beachtung oder dieser Bewegungsdrang wird als Störung sichtbar. Die gehäuften Störungen führen zu Frustration bei Lehrpersonen und Schülerinnen und Schülern bis hin zur Haltung, nicht mehr gerne lernen zu wollen.

Marcel Helbig vom Wissenschaftszentrum Berlin für Sozialforschung (WZB) betont, dass das eigentliche Problem die „Selbstüberschätzung der Jungen“ sei. Jungen glaubten, sie müssten sich nicht anstrengen, um Erfolge zu haben. Sie sehen, dass die meisten Spitzenpositionen auch so von Männern besetzt sind. Fleiß gilt unter Jungen als uncool, bei Mädchen wird es positiv akzeptiert, weil Anstrengung und Fleiß als Haltungen bei Mädchen auch später sinnvoll sind. ${ }^{27}$

Mitte der 90er Jahre wurde Koedukation kritisch reflektiert. Es wurde sichtbar, dass trotz höherer Leistungen der Mädchen beide Geschlechter die Leistung der Mädchen geringer einschätzten. Mädchen erbringen in reinen Mädchenklassen die besseren Leistungen, Jungen in gemischten Klassen. Die Benachteiligung von Mädchen in der formal koedukativen Schule führte $\mathrm{zu}$,bewusster Koedukation“28. Schule insgesamt muss in allen Aspekten ständig der geschlechterbewussten Reflexion unterzogen werden.

26 Christian Weber, Wieso Jungen schlechtere Noten bekommen. Unterschiede beim Lernen. In: Süddeutsche Zeitung vom 7.1.2013 (online abrufbar unter http://www.sueddeutsche.de/bildung/ unterschiede-beim-lernen-wieso-jungen-schlechtere-noten-bekommen-1.1566498, Lesedatum: 1. März 2015).

27 Vgl. Weber, Süddeutsche Zeitung.

$28 \mathrm{Vgl}$. Leonie Herwartz-Emden u. a. (Hg.), Mädchen in der Schule. Empirische Studien zu Heterogenität in monoedukativen und koedukativen Kontexten. Leverkusen (Budrich) 2010. Vgl. zur bewussten Koedukation exemplarisch: Landesschulrat für Vorarlberg (Hg.), Bewußte Koedukation. Anregungen zum gemeinsamen Unterricht von Mädchen und Buben. Bregenz 1997; Bundesministerium für Unterricht und kulturelle Angelegenheiten (Hg.), Aus der Rolle fallen. Praxishilfen für eine geschlechtsspezifische Pädagogik in der Schule. Wien 1995. 


\section{Doing Gender: Religionsunterricht geschlechtergerecht verändern}

Religionsunterricht ist - wie jedes schulische Lernen - gendersensibel, genderreflektierend und gendergerecht. Alle Aspekte, die für eine gendergerechte Schule wichtig sind, haben auch für den Religionsunterricht Bedeutung.

\subsection{Wahrnehmen - den Blick schärfen}

Ein solches gendersensibles, genderreflektierendes und gendergerechtes Arbeiten in den Schulen beginnt mit der Ausrichtung der eigenen Wahrnehmung und dem eigenen Bewusstsein. Dies kann geschult werden, indem z. B. immer wieder Nachrichtensendungen unter dem Aspekt gehört werden, was die jeweilige Meldung für davon betroffene Frauen (und Kinder) bedeutet. Wahrnehmung wird geschult, indem die eigenen, manchmal auch ambivalenten Bilder bzgl. Männern und Frauen ebenso reflektiert werden wie das Erleben des „eigenen“ und ,anderen“ Geschlechts, indem Gottesbilder und religiöse Erfahrungen unter dem Aspekt von Gender reflektiert werden, oder indem spezielle Dokumentationen, wie z. B. „Ich bin Sophia“ über ein Transgenderkind ${ }^{29}$ den Perspektivwechsel ermöglichen.

Da gegenwärtig eine Nivellierung der Geschlechterdifferenz und zugleich das Fortbestehen von Stereotypen sichtbar sind ${ }^{30}$, bleibt die Schulung der GenderWahrnehmung als Lehrperson wichtig: Wie verhalten sich Jungen und Mädchen in der Klasse? Welche Erwartungen habe ich an einen Jungen, an ein Mädchen, an Männer, an Frauen? Was wäre, wenn die Geschlechterrollen getauscht werden? Was wäre anders, wenn ich ein Mann, eine Frau wäre? Was müsste ich mehr, was weniger? Worum würde ich mich mehr kümmern, worum weniger? Und warum eigentlich? Natürlich können auch Rollen-Wechsel-Spiele anregend sein. Bei sportinteressierten Gruppen ist es spannend zu sehen, wann ein Sportler oder

29 Vgl. „Ich bin Sophia!“ Leben als Transgender-Kind. Ein Film von Norbert Lübbers, aus der Reihe „Menschen hautnah“ vom 30.08.2018, WDR Doku, 30 Minuten (online abrufbar unter https://www.youtube.com/watch?v=4rFSotQ-RSo, Lesedatum: 13. September 2019).

30 Vgl. Sabine Pemsel-Maier, „Geschlechter bilden“. Schlaglichter aus religionspädagogischer und bildungswissenschaftlicher Sicht. In: Margit Eckholt (Hg.), Gender studieren. Lernprozess für Theologie und Kirche. Ostfildern (Matthias Grünewald) 22017, 121-135, 123. 
eine Sportlerin medial positiv darstellt wird. Gibt es Unterschiede zwischen den Geschlechtern? U.v.m.

\subsection{Gegendert - für Jungen}

Religionsunterricht für und mit Jungen erfordert Erwachsene, die sich auf die Jungen einlassen, sich persönlich mit ihnen auseinandersetzen und die Grenzen wahren, um die Jungen in ihrer Entwicklung zu begleiten, emotionale und soziale Bildung zu fördern, sich kritisch mit geschlechtsspezifischen Rollenerwartungen und dem eigenen „Mannsein“ auseinander zu setzen sowie Rollenwechsel einzuüben. ${ }^{31}$

\subsection{Role models für Mädchen und für Jungen}

Jungen und Mädchen entwickeln durch attraktive, lebensnahe Vorbilder stärkeres Vertrauen in ihre Fähigkeiten, sind selbstbewusster und treten klarer für ihre eigenen Wünsche ein. ${ }^{32}$ Eine umfangreiche Forschungsarbeit zu Werthaltungen bei den 14 - 16jährigen Jugendlichen zeigt deutlich, dass die nahen Vorbilder wichtig sind. ${ }^{33}$ Exemplarische Impulse können sensibilisieren: Schülerinnen und Schüler besprechen oder malen, wer für sie ein Vorbild ist? Sie schreiben einen Text zur Fragestellung: „Ich möchte sein wie ...“ Es ist auch möglich, in der eigenen Gemeinde zu erforschen, welche Straßennamen nach Männern und welche nach Frauen benannt sind. Es können Denkmäler in der eigenen Region gesucht werden, verbunden mit der Fragestellung, ob diese an Männer oder an Frauen erinnern. Auch tradierte und moderne Heilige konstruieren und inszenieren Geschlecht.

31 Vgl. Andreas Obenauer, Reli für Jungs. Didaktische Impulse für einen jungengerechten Religionsunterricht. Göttingen (Vandenhoeck und Ruprecht) 2014. Vgl. Knauth, Jungen in der Religionspädagogik, $72 \mathrm{ff}$.

32 Vgl. Hans Mendl, Modelle - Vorbilder - Leitfiguren. Lernen an außergewöhnlichen Biografien. Stuttgart (Kohlhammer) 2015.

33 Vgl. Gabriele Böheim-Galehr/Helga Kohler-Spiegel, Lebenswelten - Werthaltungen junger Menschen in Vorarlberg 2016 (= FokusBildungSchule Bd. 9). Innsbruck (Studienverlag) 2017; Gabriele Böheim-Galehr/Helga Kohler-Spiegel, Lebenswelten - Werthaltungen junger Menschen in Vorarlberg (= FokusBildungSchule Bd. 9). Innsbruck (Studienverlag) 2011. 


\subsection{Möglichkeiten im Unterricht}

Gemeinsame Themen und differenzierte thematische Arbeit im Unterricht, gemeinsame und getrennte Räume für das Lernen, Möglichkeiten zum Spielen auf dem Pausenhof und im Schulhaus selbst - es gilt, Verschiedenheiten und Grenzen zwischen den Geschlechtern zu respektieren und gleichzeitig beiden Geschlechtern unabhängig vom Geschlecht Lernangebote zu machen und Lernen zu ermöglichen. Ohne ständige Betonung der Zweigeschlechtlichkeit ist es hilfreich, immer wieder die Vielfalt auch an geschlechtlicher Identität bei Kindern und Jugendlichen zu sehen - über den eigenen Fachunterricht hinaus.

Planung und Durchführung von (Religions-)Unterricht hat immer die jeweilige Lerngruppe in den Blick zu nehmen und eigene Vorstellungen über die Schülerinnen und Schüler immer wieder zu überprüfen. So kann beispielsweise die biblische Mose-Überlieferung mit Kampf und Flucht, mit Bedrohung und Rettung für Mädchen wie Jungen im Religionsunterricht spannend sein. Die Erzählung bietet - neben den vielen im Erzählstrang angebotenen Themen - auch die Möglichkeit, sich darüber auszutauschen, ob das Leben von Mose anders verlaufen wäre, wenn er ein Mädchen gewesen wäre, und was anders gewesen wäre.

Wie komplex „Doing Gender“ in der Schule ist, kann am Beispiel der geschlechtsspezifischen Leseforschung kurz gezeigt werden. Dies ist auch wegen der Bedeutung biblischer Texte im Religionsunterricht interessant: Während beim „informatorischen Lesen“ Mädchen wie Jungen etwa gleich gut sind, schneiden beim „reflektierenden Lesen“ Mädchen deutlich besser ab. Als Erklärung wird vermutet: Die frühen Szenen der Lesesozialisation sind häufig verbunden mit Vorlesesituationen mit Mutter oder Großmutter, Lesen lernen wird ,als eine weiblich konnotierte kulturelle Praxis“34 erlebt. Während entwicklungspsychologisch Mädchen stärker in der Kontinuität zur ersten (meist mütterlichen) Bezugsperson bleiben $^{35}$, erleben Jungen eher Abgrenzung und teilweise auch einen Bruch in der Identifikation mit der Mutter - und damit auch mit den mit der Mutter verbundenen Handlungen. Solange also Lesen (und Schreiben, aber auch Religion und Glaube) als weiblich konnotierte Tätigkeit angesehen wird, wird Lesen (und Schreiben und Religion und Glaube) „uncool“ sein für Jungen, die gerade dabei

34 Silvia Arzt, Bibel lesen als Mädchen, als Junge. In: Annabelle Pithan u.a. (Hg.), Gender Religion - Bildung. Beiträge zu einer Religionspädagogik der Vielfalt. Gütersloh (Gütersloher Verlagshaus) 2009, 262-272, 265.

35 Vgl. Sandra Büchel-Thalmaier, Dekonstruktive und Rekonstruktive Perspektiven auf Identität und Geschlecht. Eine feministisch-religionspädagogische Analyse (Theologische Frauenforschung in Europa, 19). Münster (LIT) 2005. 
sind, dem mütterlichen Einflussbereich zu entkommen und ihre männliche Identität zu entwickeln. ${ }^{36}$

Genderbewusstsein der Erwachsenen ist Voraussetzung für gendergerechtes und gendersensibles Arbeiten, das Geschlecht prägt mit, wie wir die Welt wahrnehmen und verstehen. Konkrete Fragen und Praxisideen für geschlechtersensible Didaktik unterstützen Bewusstmachung, Reflexion und Perspektivwechsel: Wie werden Frauen und Männer in einem Bibeltext oder einer Geschichte dargestellt? Welche Stereotypen gibt es bzgl. des Geschlechts im Text und in den Strukturen des Textes? Welche Zuschreibungen und welche Wertungen werden bzgl. der Geschlechter vorgenommen? Was verändert sich, wenn die Hauptfigur in einer Geschichte im Geschlecht verändert wird und die Szene aus einer anderen Geschlechter-Perspektive erzählt oder gespielt wird? Es schafft Bewusstsein, wenn beispielsweise beim Erzählen biblischer Überlieferung die Figuren im Geschlecht verändert werden, um so geschlechtsstereotype Zuschreibungen von Verhalten und Eigenschaften bewusst zu machen.

Für die konkrete Arbeit empfiehlt Faulstich-Wieland einen Dreischritt: „Dramatisierung - Reflexion - Entdramatisierung“. Um die Kategorie Geschlecht bewusst in den Blick zu nehmen, ist es in einem ersten Schritt oft notwendig, „Geschlecht zunächst zu dramatisieren, Differenzen herauszustellen, alltägliche doing gender Situationen zu entlarven“37. Darauf folgt als zweiter Schritt die Reflexion, wo beispielsweise Geschlechts-Differenzen liegen und womit sie sich begründen oder erklären. Erst danach ist es möglich zu entdramatisieren, also die Heterogenität, Kompetenzen und Defizite der individuellen Schülerinnen und Schüler und nicht geschlechtliche Zuordnungen in den Blick zu nehmen.

\section{Schluss}

Gendergerechtes Arbeiten im Religionsunterricht ist mit der Entscheidung der Lehrperson verbunden, dies zu tun. „Doing Gender“ macht bewusst, dass Geschlechterverhältnis und Geschlechterdifferenz, Geschlechtszugehörigkeit und

36 Vgl. Arzt, Bibel lesen, $265 \mathrm{f}$.

37 Vgl. Hannelore Faulstich-Wieland u. a., Einleitung: Das Projekt Genus. In: Hannelore Faulstich-Wieland u. a. (Hg.), Genus - geschlechtergerechter naturwissenschaftlicher Unterricht in der Sekundarstufe 1. Bad Heilbrunn (Julius Klinkhardt) 2008, 9-15, 11. Vgl. Leonie Herwartz-Emden u. a., Mädchen und Jungen in Schule und Unterricht. Stuttgart (Kohlhammer) 2012, $71 \mathrm{f}$ und $83 \mathrm{f}$. Vgl. Regine Gildemeister, Doing Gender: Soziale Praktiken der Geschlechterunterscheidung. In: Ruth Becker/Beate Kortendiek (Hg.), Handbuch Frauen- und Geschlechterforschung. Theorie, Methoden, Empirie. Wiesbaden (Springer) ${ }^{32010,137-145,137 .}$ 
Geschlechtsidentität nicht festgeschrieben sind, sondern in Interaktionen immer wieder neu hergestellt werden. Insofern muss „Doing Gender“ auf der Ebene von Wahrnehmung und Bewusstsein präsent sein, um entlang der Interaktionen, der Inhalte und der Methoden im Religionsunterricht präsent zu sein.

Und es bleibt zu erinnern: Unter dem Titel „Über die Unmöglichkeit, Politik durch Pädagogik zu ersetzen“ haben die Autoren Hamburger, Seus und Wolter bereits 1981 (im Kontext Interkultureller Pädagogik) vor der Verwechslung der Aufgaben gewarnt ${ }^{38}$, dies gilt auch für unser Thema. Auch geschlechtersensible pädagogische Arbeit muss einhergehen mit der Veränderung geschlechterbezogener Ungerechtigkeiten, vordefinierter Geschlechterverhältnisse und gesellschaftlich festgelegter Rollenbilder.

38 Vgl. Franz Hamburger u. a., Über die Unmöglichkeit, Politik durch Pädagogik zu ersetzen. In: Unterrichtswissenschaft (1981) Nr. 2, 158-167. 